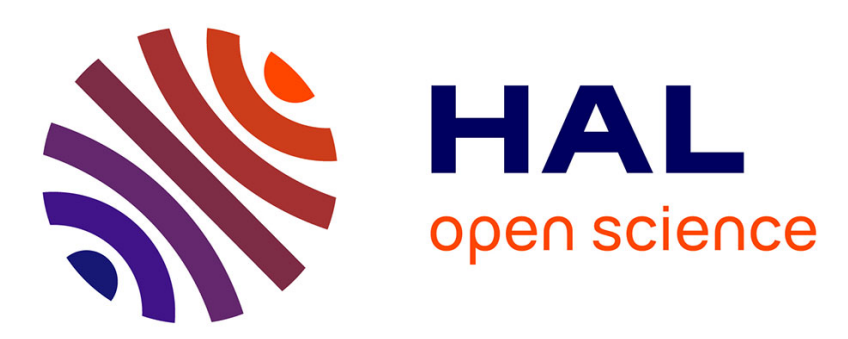

\title{
Sparse Supernodal Solver Using Block Low-Rank Compression
}

Grégoire Pichon, Eric Darve, Mathieu Faverge, Pierre Ramet, Jean Roman

\section{To cite this version:}

Grégoire Pichon, Eric Darve, Mathieu Faverge, Pierre Ramet, Jean Roman. Sparse Supernodal Solver Using Block Low-Rank Compression. [Research Report] RR-9022, Inria Bordeaux Sud-Ouest. 2017, pp.24. hal-01450732v3

\section{HAL Id: hal-01450732 https://hal.inria.fr/hal-01450732v3}

Submitted on 1 Feb 2017

HAL is a multi-disciplinary open access archive for the deposit and dissemination of scientific research documents, whether they are published or not. The documents may come from teaching and research institutions in France or abroad, or from public or private research centers.
L'archive ouverte pluridisciplinaire HAL, est destinée au dépôt et à la diffusion de documents scientifiques de niveau recherche, publiés ou non, émanant des établissements d'enseignement et de recherche français ou étrangers, des laboratoires publics ou privés. 


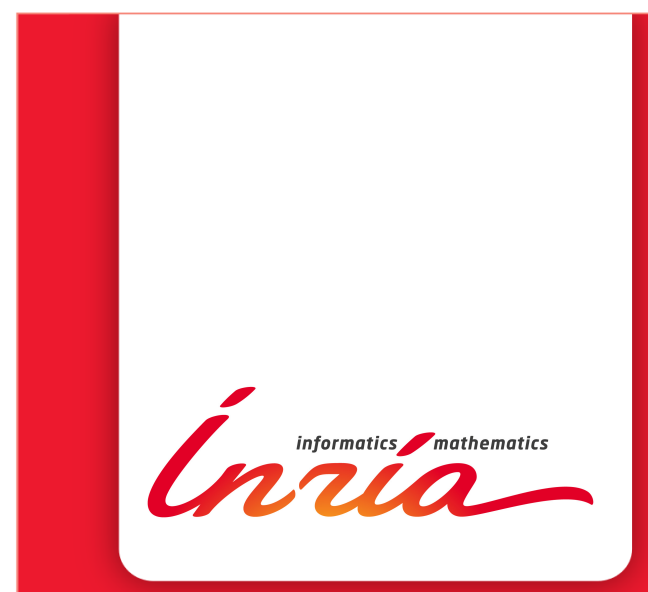

\section{Sparse Supernodal Solver Using Block Low-Rank Compression}

Grégoire Pichon, Eric Darve, Mathieu Faverge, Pierre Ramet, Jean Roman

\section{RESEARCH}

\section{REPORT}

$\mathrm{N}^{\circ} 9022$

January 2017

Project-Team HiePACS 



\title{
Inzián
}

\section{Sparse Supernodal Solver Using Block Low-Rank Compression}

\author{
Grégoire Pichon㖄, Eric Darve $\S$, Mathieu Faverge $\mathbb{\mathbb { T }}^{\ddagger}$, Pierre \\ Ramet $^{\dagger * \ddagger}$, Jean Roman $* \mathbb{I \ddagger}$ \\ Project-Team HiePACS \\ Research Report nº 9022 - January 2017 - 22 pages
}

\begin{abstract}
This paper presents two approaches using a Block Low-Rank (BLR) compression technique to reduce the memory footprint and/or the time-to-solution of the sparse supernodal solver PaStiX. This flat, non-hierarchical, compression method allows to take advantage of the low-rank property of the blocks appearing during the factorization of sparse linear systems, which come from the discretization of partial differential equations. The first approach, called Minimal Memory, illustrates the maximum memory gain that can be obtained with the BLR compression method, while the second approach, called Just-In-Time, mainly focuses on reducing the computational complexity and thus the time-to-solution. Singular Value Decomposition (SVD) and Rank-Revealing QR (RRQR), as compression kernels, are both compared in terms of factorization time, memory consumption, as well as numerical properties. Experiments on a single node with 24 threads and $128 \mathrm{~GB}$ of memory are presented on a set of matrices from real-life problems. We demonstrate a memory footprint reduction of up to 4.4 times using the Minimal Memory strategy and a computational time speedup of up to 3.3 times with the Just-In-Time strategy.
\end{abstract}

Key-words: Sparse linear solver, block low-rank compression, PAStiX direct solver, multithreaded architectures

* Inria Bordeaux - Sud-Ouest, Talence, France

$\dagger$ University of Bordeaux, Talence, France

$\ddagger$ CNRS (Labri UMR 5800), Talence, France

$\S$ Mechanical Engineering Department, Stanford University, United States

$\mathbb{I}$ Bordeaux INP, Talence, France

RESEARCH CENTRE

BORDEAUX - SUD-OUEST

200 avenue de la Vieille Tour

33405 Talence Cedex 


\section{Un solveur supernodal creux utilisant une compression de rang faible par bloc}

Résumé : Ce papier présente deux approches utilisant les techniques de compression de rang faible par bloc (BLR) afin de réduire l'empreinte mémoire et/ou le temps de résolution du solveur superndal PASTIX. Cette technique de compression à plat, non hiérarchique, permet de tirer parti des propriétés de rang faible dans les blocs obtenus lors de la factorisation du système linéaire provenant par exemple de la discrétisation des équations différentielles partielles. La première approche, appelée Minimal Memory, montre le gain mémoire maximum qu'il est possible d'obtenir avec une compression BLR, alors que la seconde approche, appelée Just-InTime, se concentre principalement sur la réduction du temps de calcul pour la résolution du système. Dans cette étude, nous comparons, en termes de temps de calcul et de consommation mémoire, les noyaux de compression qui utilisent soit la technique de décomposition en valeurs propres singulières (SVD) soit la factorisation QR avec détermination du rang (RRQR). Nous avons réalisé les expériences sur un noeud composé de 24 coeurs avec 128 GB de mémoire sur une collection de matrices issues d'applications réelles. Nous montrons que la consommation mémoire peut-être réduite jusqu'à un facteur 4.4 avec la stratégie Minimal Memory et que le temps de calcul peut être divisé par 3.3 en utilisant la stratégie Just-In-Time.

Mots-clés : Solveur linéaire creux, compression de rang faible par bloc, PASTiX solveur direct, architectures multi-thread 


\section{Introduction}

Many scientific applications such as electromagnetism, geophysics or computational fluid dynamics use numerical models that require to solve linear systems of the form $A x=b$, where the matrix $A$ is sparse and large. In order to solve these problems, a classic approach is to use a sparse direct solver which factorizes the matrix into a product of triangular matrices before solving triangular systems.

Yet, there are still limitations to solve larger and larger systems in a black-box approach without any knowledge of the geometry of the underlying partial differential equation. Memory requirements and time-to-solution limit the use of direct methods for very large matrices. On the other hand, for iterative solvers, general black-box preconditioners that can ensure fast convergence for a wide range of problems are still missing.

In the context of sparse direct solvers, some recent works have investigated the low-rank representations of dense blocks appearing during the sparse matrices factorization, by compressing blocks through many possible compression formats such as Block Low-Rank (BLR), $\mathcal{H}, \mathcal{H}^{2}$, HSS, HODLR. . . These different approaches allow a reduction of the memory requirement and/or the time to solution. Depending on the compression strategy, solvers require knowledge of the underlying geometry to tackle the problem or can do it in a purely algebraic fashion.

Hackbusch [1] introduced the $\mathcal{H}$-LU factorization for dense matrices, which compresses the matrix into a hierarchical matrix before applying low-rank operations instead of classic dense operations. In the same paper, an extension of the dense version was designed for sparse matrices using nested dissection ordering. In [2], $\mathcal{H}$-LU factorization is used in an algebraic context. Performance, as well as a comparison of $\mathcal{H}$-LU with some sparse direct solvers is presented in 3 . Kriemann [4 implemented this algorithm using Direct Acyclic Graphs.

The Hierarchically Off-Diagonal Low-Rank (HODLR) compression technique was used in a multifrontal sparse direct solver in $[5$ to accelerate the elimination of large fronts. It was fully extended for a sparse purpose in $[6]$ and uses Boundary Distance Low-Rank (BDLR) to allow both time and memory savings. A supernodal solver using a compression technique close to HODLR was presented in 7. The proposed approach allows memory savings and can be faster than standard preconditioned techniques. However, it is slower than the direct approach in the benchmarks and requires an estimation of the rank to use randomized techniques and accelerate the solver.

There have been different works around the use of Hierarchically Semi-Separable (HSS) matrices in sparse direct solvers. In [8], Xia et al. presented a solver for 2D geometric problems, where all operations are realized algebraically. In [9], a geometric solver was developed, but contribution blocks are not compressed, making memory savings impossible. [10] proposed an algebraic code that uses randomized sampling to manage low-rank blocks and to allow memory savings.

$\mathcal{H}^{2}$ arithmetic has also been applied to sparse solvers. In 11 , a fast sparse $\mathcal{H}^{2}$ solver, called LoRaSp, based on extended sparsification was introduced. In [12, a variant of LoRaSp, aimed at improving the quality of the solver when used as a preconditioner, was presented, as well as a numerical analysis of the convergence with $\mathcal{H}^{2}$ preconditioning. In particular, this variant was shown to lead to a bounded number of iterations irrespective of problem size and condition number (under certain assumptions). In $[13$ a fast sparse solver was introduced based on interpolative decomposition and skeletonization. It was optimized for meshes that are perturbations of a structured grid. In [14], an $\mathcal{H}^{2}$ sparse algorithm was described. It is similar in many respects to [11, and extends the work of [13. All these solvers have a guaranteed linear complexity, for a given error tolerance, and assuming a bounded rank for all well-separated pairs of clusters (the admissibility criterion in Hackbusch et al.'s terminology). 
Block Low-Rank compression have also been investigated for dense matrices [15], and for sparse linear systems considering a multifrontal method [16. Considering that these approaches are close to the current study, a detailed comparison will be described in Section 5 .

The first objective of this work is to combine a generic sparse direct solver with recent work on matrix compression to come up with a way to solve larger problems, overcoming the memory limitations and accelerating the time-to-solution. The second objective is to keep the blackbox algebraic approach of sparse direct solvers, by relying on methods that are independent of the underlying problem geometry. In this paper, we consider the multi-threaded sparse direct solver PASTIX [17] and we introduce a BLR compression strategy to reduce its memory and computational cost. We developed two strategies: Minimal Memory, which focuses on reducing the memory consumption, and Just-In-Time which focuses on reducing the time-to-solution (factorization and solve steps).

During the factorization, the first strategy compresses the sparse matrix from the beginning and exploits complicated low-rank numerical operations to keep the memory cost of the factorized matrix as low as possible. The second one compresses the information as late as possible to avoid the cost of low-rank update operations. The resulting solver can be used either as a direct solver for low accuracy solutions or as a high-accuracy preconditioner for iterative methods, requiring only a few iterations to reach machine precision.

In Section 1, we go over basic aspects of sparse supernodal direct solvers. The two strategies, introduced in PASTIX, are then presented in Section 2, before detailing low-rank kernels in Section 3 In Section 4 we perform experiments comparing the two BLR strategies with the original approach - that uses only dense blocks - in terms of memory consumption, timeto-solution and numerical behavior. Section 5 surveys in more details related works on BLR for dense and/or sparse direct solvers, highlighting the differences with our approach, before discussing how to extend this work to a hierarchical format (H, HSS, HODLR...).

\section{Background}

The common approach used by direct solvers is composed of four main steps: 1) ordering of the unknowns, 2) computation of a symbolic block structure, 3) numerical block factorization, and 4) triangular systems solves. In the rest of the paper, we focus on problems leading to sparse systems with a symmetric pattern.

The purpose of the first step is to minimize the fill-in — zeros becoming non-zeros during factorization - that occurs during the numerical factorization to reduce the number of operations as well as the memory requirements to solve the problem. In order to both reduce fill-in and exhibit parallelism, the nested dissection [18] algorithm is widely used through libraries such as Metis [19] or Sсотсн [20]. Each set of vertices corresponding to a separator constructed during the nested dissection is called a supernode.

From the resulting supernodal partition, the second step predicts the symbolic block structure of the final factorized matrix $(L)$ and the block elimination tree. This block structure is composed of one block of columns (column block) for each supernode of the partition, with a dense diagonal block and several dense off-diagonal blocks, as presented in Figure 1 for a 3D Laplacian.

The goal is to exhibit large block structures to leverage efficient Level 3 BLAS kernels during the numerical factorization. However, one may notice (cf. Figure 1) that the symbolic structure obtained with a general partitioning tool might be composed of many small off-diagonal blocks contributing to larger blocks. These off-diagonal blocks might be grouped together by adding zeros to the structure if the BLAS efficiency gain is worthwhile and if the memory overhead induced by the fill-in is limited. Alternatively, it is also possible to reorder supernode unknowns 


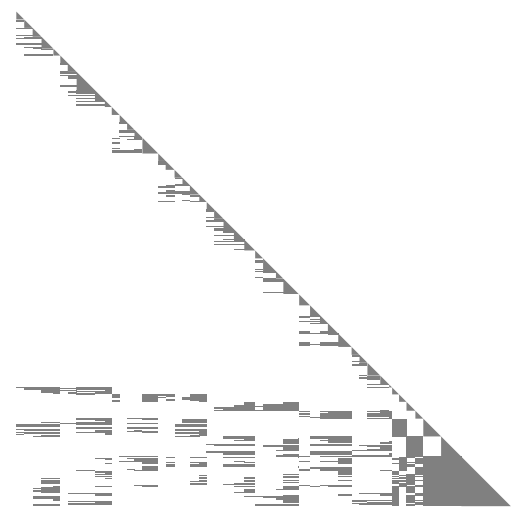

Figure 1: Symbolic factorization of a $10 \times 10 \times 10$ Laplacian partitioned using Scotch.

to group off-diagonal blocks together without additional fill-in. A traveler salesman strategy is implemented in PASTIX 21 and divides by more than two the number of off-diagonal blocks. Other approaches like [10,16] perform a $k$-way ordering of supernodes, starting from a reconnected graph of a separator, to order consecutively vertices belonging to a same local part of the separator's graph. Such re-ordering technique also allows to reduce ranks of the low-rank blocks as shown in [16. To introduce more parallelism and data locality, the final structure can then be split in tiles as it is now commonly done in dense linear algebra libraries. These first two steps of direct solvers are preprocessing stages independent from numerical values. Note that these steps can be computed once to solve multiple problems similar in structure but with different numerical values.

Finally, the last two steps, numerical factorization and triangular systems solves, perform the numerical operations. We consider here only the first one for the PAStiX solver. During the numerical factorization, the elimination of each supernode (column block) is similar to standard dense algorithms: 1) factorize the dense diagonal block, 2) solve the off-diagonal blocks belonging to this supernode, and 3) apply the updates on the trailing submatrix (cf. Section 2).

\section{Block Low-Rank solver}

In this section, we describe the main contribution of this paper which is a BLR solver developed within the PAStiX library. First we introduce the notations used in this article, and the basics used to integrate low-rank blocks in the solver. Then, using the newly introduced structure, we describe two different strategies leading to a sparse direct solver that optimizes the memory consumption or the time-to-solution.

\section{$2.1 \quad$ Notations}

Let us consider the symbolic block structure of a factorized matrix $L$, obtained throught the symbolic block factorization. Initially, we allocate this structure initialized with the entries of $A$ and perform an in-place factorization. We denote initial blocks $A$ and when a block corresponds to its final state, it becomes $L$ (or $U$ ). The matrix is composed of $N_{c b l k}$ column blocks, where each column block is associated to a supernode, or to a subset of unknowns in a supernode when the later is split to create parallelism. Each column block $k$ is composed of $b_{k}+1$ blocks, as presented in Figure 2 where: 


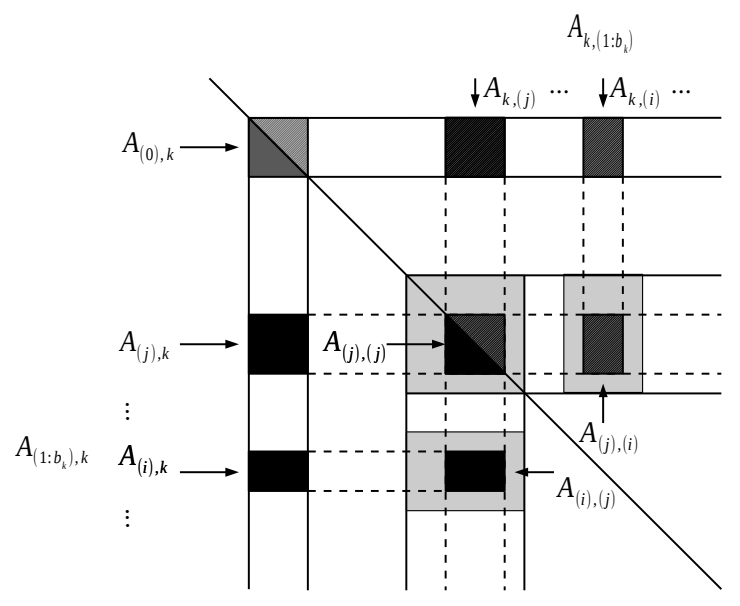

Figure 2: Symbolic block structure and notations used for the algorithms for one column block $k$, and its associated blocks.

- $A_{(0), k}\left(=A_{k,(0)}\right)$ is the dense diagonal block;

- $A_{(j), k}$ is the $j^{\text {th }}$ off-diagonal block in the column block with $1 \leq j \leq b_{k},(j)$ being a multi-index describing the row interval of each block, and respectively, $A_{k,(j)}$ is the $j^{\text {th }}$ off-diagonal block in the row block;

- $A_{\left(1: b_{k}\right), k}$ represents all the off-diagonal blocks of the column block $k$, and $A_{k,\left(1: b_{k}\right)}$ all the off-diagonal blocks of the symmetric row block;

- $A_{(i),(j)}$ is the rectangular dense block corresponding to the rows of the multi-index $(i)$ and to the columns of the multi-index $(j)$.

In addition, we denote $\hat{A}$ the compressed representation of a matrix $A$.

\subsection{Sparse direct solver using BLR compression}

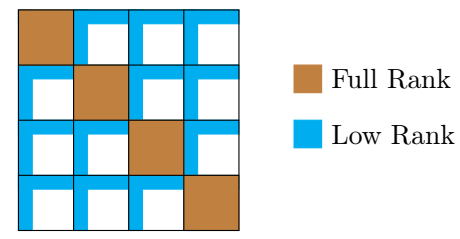

Figure 3: Block Low-Rank compression.

The BLR compression scheme is a flat, non-hierarchical format, unlike others mentioned in the introduction. If we consider the example of a dense matrix, the BLR format clusters the matrix into a set of smaller blocks, as presented in Figure 3 Diagonal blocks are kept dense and off-diagonal blocks, which represent long distance interactions in the graph, are low-rank. Thus, these off-diagonal blocks can be represented through a low-rank form $u v^{t}$, obtained with a compression technique such as Singular Value Decomposition (SVD) or Rank-Revealing QR (RRQR) factorization. Compression techniques are detailed in Section 3. 
We propose in this paper to similarly apply this scheme to the symbolic block structure of sparse direct solvers. First, diagonal blocks of the largest supernodes in the block elimination tree can be considered as large dense matrices which are compressible with the BLR approach. In fact, as we have seen previously, it is common to split these supernodes into a set of smaller column blocks in order to increase the level of parallelism. Thus, the block structure resulting from this operation gives the cluster of the BLR compression format. Second, interaction blocks from two large supernodes are by definition long distance interactions, and thus can be represented by a low-rank form. It is then natural to store them as low-rank blocks as long as they are large enough. To summarize, if we take the final symbolic block structure (after splitting) used by the PASTIX solver, all diagonal blocks are considered dense, and all off-diagonal blocks might be stored using a low-rank structure. In practice, we limit this compression to blocks of a minimal size, and all blocks with high ranks are kept dense.

Relying on the original block structure, adapting the solver to block low-rank compression mainly relies on the replacement of the dense operations with the equivalent low-rank operations. Still, different variants of the final algorithm can be obtained by changing when and how the lowrank compression is applied. We introduce two scenarios: Minimal Memory, which compresses the blocks before any other operations, and Just-In-Time which compresses the blocks after they received all their contributions.

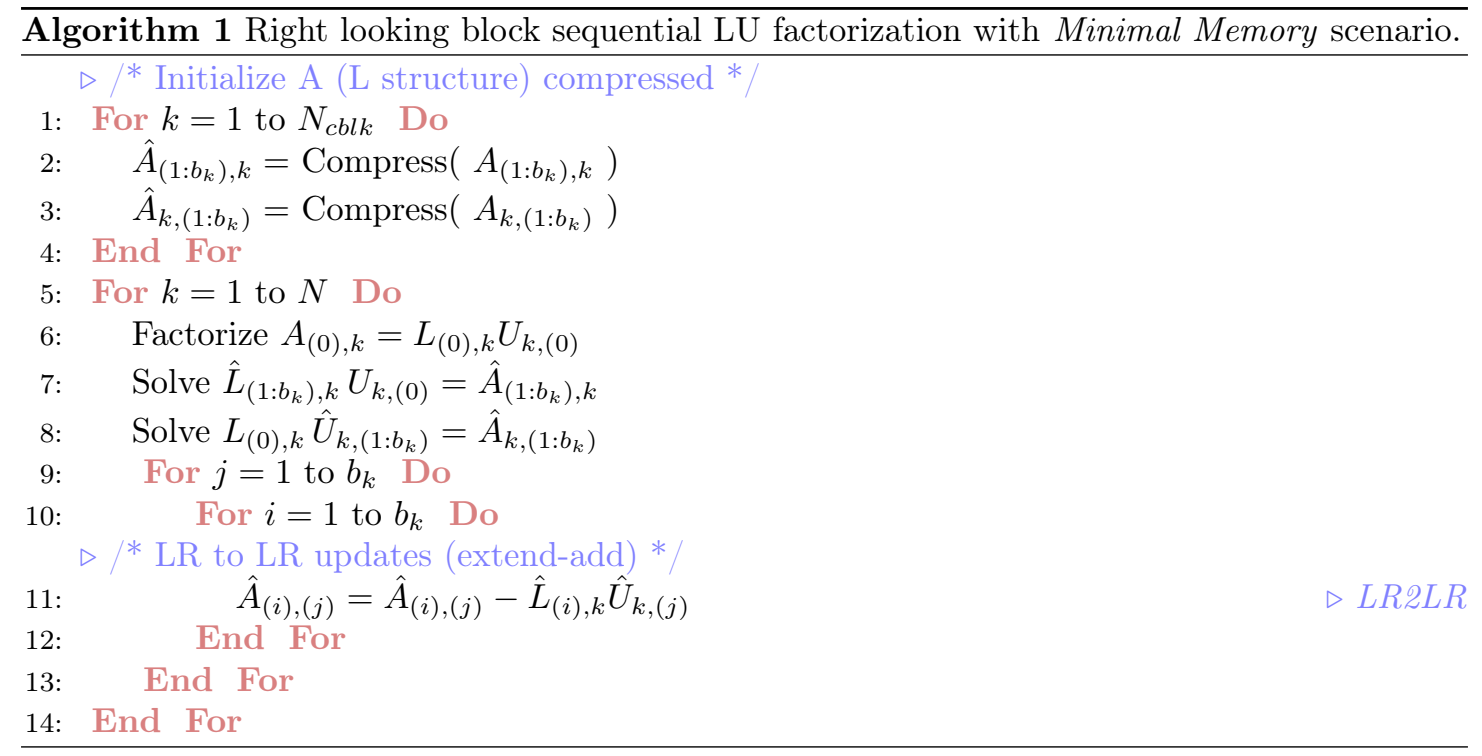

\subsubsection{Minimal Memory}

This scenario, described by Algorithm 1, starts by compressing the original matrix $A$. Thus, all low-rank blocks that are large enough are compressed directly from the original sparse form to the low-rank representation (lines $1-4$ ). Note that for a matter of conciseness, loops of compression and solve over all off-diagonal blocks are merged into a single operation. In this scenario, compression kernels and later operations could have been performed on a sparse format, such as CSC for instance, until we get some fill-in. However, for the sake of simplicity we use a low-rank form throughout the entire algorithm to rely on blocks and not just on sets of values. Then, each classic dense operation on a low-rank block is replaced by a similar kernel operating on 
low-rank forms, even for the usual matrix-matrix multiplication (GEMM) kernel that is replaced by the equivalent LR2LR kernel operating on three low-rank matrices (cf. Section 3).

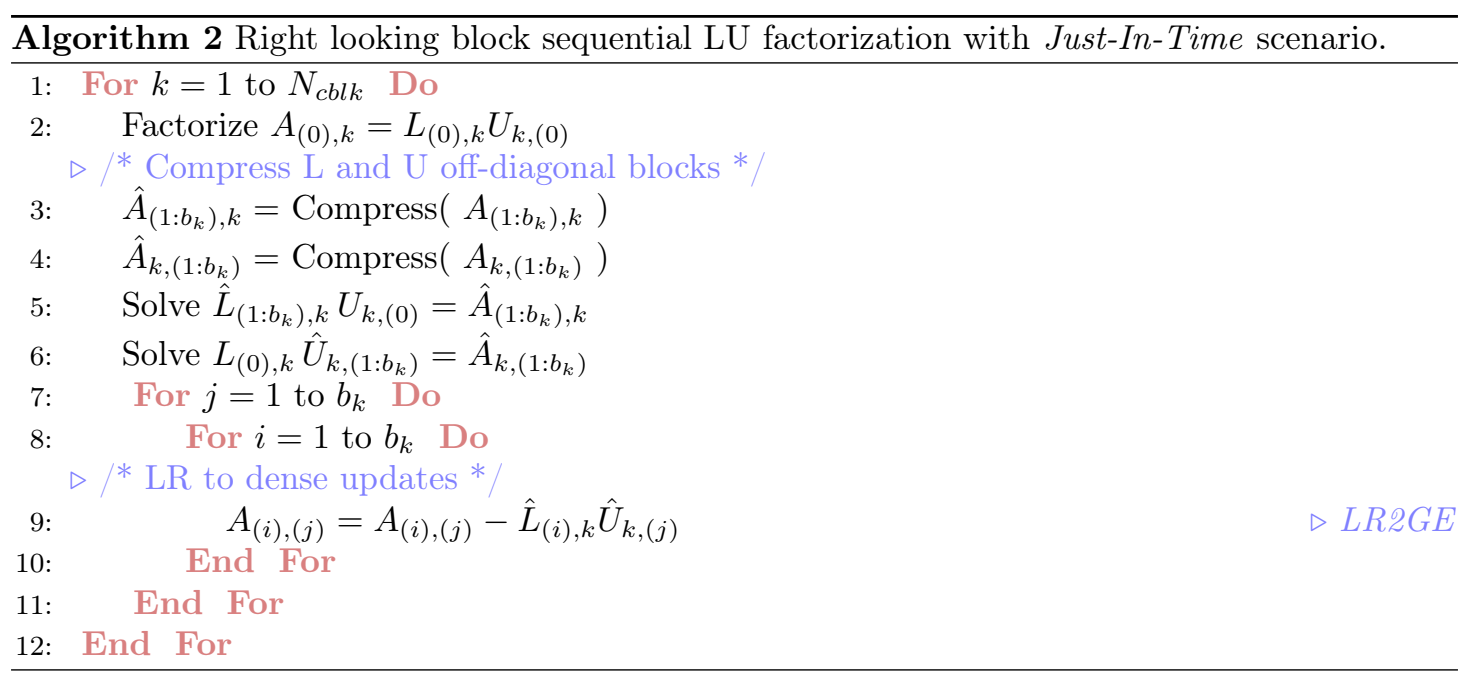

\subsubsection{Just-In-Time}

This second scenario, described by Algorithm 2 delays the compression of each supernode after all contributions have been accumulated. The algorithm is thus really close to the previous one with the only difference being in the update kernel, LR2GE, at line 9, which accumulates contributions on a dense block, and not on a low-rank form.

This operation, as we described in Section 3, is much simpler than the LR2LR kernel, and is faster than a classic GEMM. However, by compressing the initial matrix $A$, and maintaining the low-rank structure throughout the factorization with the LR2LR kernel, Minimal Memory can reduce more drastically the memory footprint of the solver. Indeed, the final dense structure of the factorized matrix is never allocated, as opposed to Just-In-Time that requires it to accumulate the contributions. The final matrix is compressed with similar sizes in both scenarios.

\section{Low-rank kernels}

We introduce in this section the low-rank kernels used to replace the dense operations, and we present a complexity study of these kernels. Two families of operations are studied to reveal the rank of a matrix: Singular Value Decomposition (SVD) which leads to smaller ranks, and Rank-Revealing QR (RRQR) which allows a faster implementation.

\subsection{Compression}

The goal of low-rank compression is to represent a general dense matrix $A$ of size $m_{A}$-by- $n_{A}$ by its compressed version $\hat{A}=u_{A} v_{A}^{t}$, where $u_{A}$, and $v_{A}$, are respectively matrices of size $m_{A}$-by- $r_{A}$, and $n_{A}$-by- $r_{A}$, with $r_{A}$ being the rank of the block supposed small with respect to $m_{A}$ and $n_{A}$. In order to keep a given numerical accuracy we have to choose $r_{A}$ such that $\|A-\hat{A}\| \leq \tau\|A\|$, where $\tau$ is the prescribed tolerance. 


\subsubsection{SVD}

$A$ is decomposed as $U \sigma V^{t}$. The low-rank form of $A$ is thus made out of the first $r_{A}$ singular values and their associated singular vectors such that: $\sigma_{r_{A}+1} \leq \tau, u_{A}=U_{r_{A}}$, and $v_{A}^{t}=\sigma_{1: r_{A}} V_{r_{A}}^{t}$ with $U_{r_{A}}$ being the first $r_{A}$ columns of $U$, and respectively for $V$. This process requires $\Theta\left(m_{A}^{2} n_{A}+\right.$ $n_{A}^{2} m_{A}+n_{A}^{3}$ ) operations.

\subsubsection{RRQR}

$A$ is decomposed as $P Q R$, where $P$ is a permutation matrix, and $Q R$ the $\mathrm{QR}$ decomposition of $P^{-1} A$. The rank- $r_{A}$ form of $A$ is then formed by $u_{A}=Q_{r_{A}}$, the first $r_{A}$ columns of $Q$, and $v_{A}^{t}=R_{r_{A}}$, the first $r_{A}$ rows of $R$. The main advantage of this process is that it can stop the factorization as soon as the norm of the trailing submatrix $\tilde{A}_{\left(r_{A}+1: m_{A}, r_{A}+1: n_{A}\right)}=A-P Q_{r_{A}} R_{r_{A}}$ is lower than $\tau$. Thus, the complexity is lowered to $\Theta\left(n_{A} r_{A}^{2}\right)$ operations.

SVD compression is much more expensive than RRQR. However, for a given tolerance, SVD returns lower ranks. Put another way, for a given rank, SVD will have a better numerical accuracy. Thus, there is a trade-off between time-to-solution (RRQR) versus memory consumption and numerical accuracy (SVD).

Note that for the Minimal Memory scenario, the first compression (of sparse blocks) may be realized using Lanczos's methods, to take advantage of sparsity. However, both SVD and RRQR algorithms take inherently advantage of these zeros. In addition, most of the low-rank compressions are applied to blocks stored as dense blocks and it represents the main part of the computations.

\subsection{Solve}

The solve operation for a generic lower triangular matrix $L$ is applied to blocks in low-rank forms in our two scenarios: $L \hat{x}=\hat{b} \Leftrightarrow L u_{x} v_{x}^{t}=u_{b} v_{b}^{t}$. Then, with $v_{x}^{t}=v_{b}^{t}$, the operation is equivalent to apply a dense solve only to $u_{b}^{t}$, and the complexity is only $\Theta\left(m_{L}^{2} r_{x}\right)$, instead of $\Theta\left(m_{L}^{2} n_{L}\right)$ for the dense representation.

\subsection{Update}

Let us consider the generic update operation, $C=C-A B^{t}$. Note that the PAStiX solver stores $L$, and $U^{t}$ if required. Then, the same update is performed for Cholesky and LU factorizations. We break the operation in two steps: the product of two low-rank blocks, and the addition of a low-rank block and either a dense block $(L R 2 G E)$, or a low-rank block $(L R 2 L R)$.

\subsubsection{Low-rank matrices product}

This operation can simply be expressed as two dense matrix products: $\hat{A} \hat{B}^{t}=\left(u_{A}\left(v_{A}^{t} v_{B}\right)\right) u_{B}^{t}=$ $u_{A}\left(\left(v_{A}^{t} v_{B}\right) u_{B}^{t}\right)$ where $u_{A}$ is kept unchanged if $r_{A} \leq r_{B}\left(u_{B}^{t}\right.$ is kept otherwise) to lower the complexity.

However, it has been shown in 15] that the rank $r_{A B}$ of the product of two low-rank matrices of ranks $r_{A}$ and $r_{B}$ is usually smaller than $\min \left(r_{A}, r_{B}\right)$. Moreover, $u_{A}$ and $u_{B}$ are both orthogonal, so the matrix $T=\left(v_{A}^{t} v_{B}\right)$ has the same rank as $\hat{A} \hat{B}^{t}$. Thus, the complexity can be further reduced by transforming the matrix product to the following series of operations: 


$$
\begin{aligned}
T & =v_{A}^{t} v_{B} \\
\hat{T}=\widehat{v_{A}^{t} v_{B}} & =u_{T} v_{T}^{t} \\
u_{A B} & =u_{A} u_{T} \\
v_{A B}^{t} & =v_{T}^{t} v_{B}^{t} .
\end{aligned}
$$

\subsubsection{Low-rank matrices addition}

Let us consider the next generic operation $C^{\prime}=C-u_{A B} v_{A B}^{t}$, with $m_{A B} \leq m_{C}$ and $n_{A B} \leq n_{C}$ as it generally happens in supernodal methods. This is illustrated for example by the update block $A_{(i),(j)}$ in Figure 2 ,

If $C$ is not compressed as in the LR2GE kernel, $C^{\prime}$ will be dense too, and the addition of the two matrices is nothing else than a GEMM kernel. The complexity of this operation grows as $\Theta\left(m_{A B} n_{A B} r_{A B}\right)$.

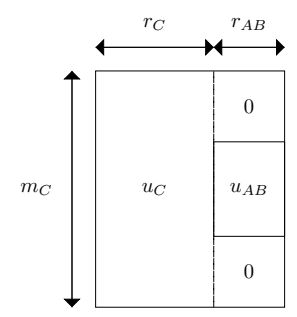

Figure 4: Accumulation of two low-rank matrices when sizes do not match.

If $C$ is compressed as in the LR2LR kernel, $C^{\prime}$ will be compressed too, and

$$
\begin{array}{r}
\hat{C}^{\prime}=u_{C} v_{C}^{t}-u_{A B} v_{A B}^{t} \\
u_{C^{\prime}} v_{C^{\prime}}^{t}=\left[u_{C}, u_{A B}\right]\left(\left[v_{C},-v_{A B}\right]\right)^{t}
\end{array}
$$

where [,] is the concatenation operator. This is the commonly named extend-add operation. Without further optimization, this operation costs only two copies. In the case of supernodal method, adequate padding is also required to align the vectors coming from $A B$, and $C$ matrices as it is presented in Figure 4 for the $u$ vectors. The operation on $v$ is similar.

One can notice that, kept as this, the rank of the updated $C$ is now $r_{C}+r_{A B}$. When accumulating multiple updates, the rank grows quickly and the storage exceeds the original dense version. In order to maintain a small rank for $C$, recompression techniques are used. As for the compression kernel, both SVD and RRQR algorithms can be used.

Recompression using SVD it first requires to compute a QR decomposition for both composed matrices:

$$
\left[u_{C}, u_{A B}\right]=Q_{1} R_{1} \text { and }\left[v_{C},-v_{A B}\right]=Q_{2} R_{2} .
$$

Then, the temporary matrix $T=R_{1} R_{2}^{t}$ is compressed using the SVD algorithm described previously. This gives the final $\hat{C}^{\prime}$ with:

$$
u_{C^{\prime}}=\left(Q_{1} u_{T}\right) \text { and } v_{C^{\prime}}=\left(Q_{2} v_{T}\right) .
$$

The complexity of this operation is decomposed as follows: $\Theta\left(\left(m_{C}+n_{C}\right)\left(r_{C}+r_{A B}\right)^{2}\right)$ for the QR decomposition of equation (7), $\Theta\left(\left(r_{C}+r_{A B}\right)^{3}\right)$ for the SVD decomposition, and finally $\Theta\left(\left(m_{C}+n_{C}\right)\left(r_{C}+r_{A B}\right) r_{C^{\prime}}\right)$ for the application of both $Q_{1}$ and $Q_{2}$. 
Recompression using RRQR this solution takes advantage of the orthogonality of both $u_{C}$

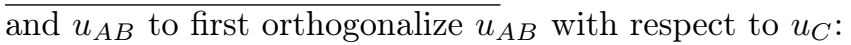

$$
u_{A B}^{*}=u_{A B}-u_{C}\left(u_{C}^{t} u_{A B}\right) .
$$

We obtain an orthonormal basis $\left[u_{C}, u_{A B}^{*}\right]$ such that:

$$
\left[u_{C}, u_{A B}\right]=\left[u_{C}, u_{A B}^{*}\right] \times\left(\begin{array}{cc}
I & u_{C}^{t} u_{A B} \\
0 & I
\end{array}\right) .
$$

We follow by applying the RRQR algorithm to:

$$
\left(\begin{array}{cc}
I & u_{C}^{t} u_{A B} \\
0 & I
\end{array}\right) \times\left(\left[v_{C}, v_{A B}\right]\right)^{t}=P Q R .
$$

As for the compression, we keep the $k=r_{C^{\prime}}$ first columns of $Q$, and rows of $R$ to form the final $C^{\prime}$ :

$$
u_{C^{\prime}}=\left(\left[u_{C}, u_{A B}^{*}\right] P Q_{k}\right) \text { and } v_{C^{\prime}}^{t}=R_{k} .
$$

Note that $u_{C^{\prime}}$ is kept orthogonal for future updates.

When the RRQR algorithm is used, the complexity of the recompression is then composed of: $\Theta\left(r_{C} r_{A B} m_{A B}\right)$ to form the intermediate product $u_{C}^{t} u_{A B}, \Theta\left(m_{C} r_{C} r_{A B}\right)$ to form the orthonormal basis, $\Theta\left(n_{A B} r_{A B} r_{C}\right)$ to generate the temporary matrix used in (11), $\Theta\left(\left(r_{C}+\right.\right.$ $\left.\left.r_{A B}\right) n_{C} r_{C^{\prime}}\right)$ to apply the RRQR algorithm, and finally again $\Theta\left(\left(r_{C}+r_{A B}\right) n_{C} r_{C^{\prime}}\right)$ to compute the final $u_{C^{\prime}}$.

\subsection{Summary}

Table 1 presents the computational complexity for the two low-rank strategies with respect to the original version of the solver. To get the main factor of the complexity, we make the assumption that $m_{C} \geq m_{A} \geq m_{B}, r_{A} \geq r_{B}, m_{C} \geq n_{C}$, and $r_{C} \leq r_{C^{\prime}}$. One can note that the JustIn-Time strategy performs the calculation of the low-rank contribution before assembling the matrix explicitly to apply a dense modification. The main factor of the complexity does not depend on $n_{A}$ but on the ranks $r_{A}$ and $r_{B}$ : there are fewer operations to be performed. On the other hand, the Minimal Memory strategy requires to use either SVD or RRQR recompression, for which the complexity depends on $m_{C}$ and $n_{C}$, the dimensions of the block $C$. It explains why this strategy is slower than the original solver.

When considering dense matrices, a low-rank matrix is usually modified by a contribution of the same size: the low-rank extend-add process may be efficient and lead to performance gain [15. It is also the case for the CUFS strategy in BLR-MUMPS, which compresses a dense front before applying operations between low-rank blocks of the same size.

In our case, a block $C$ receives many small contributions, see Figure 1 as stated by the separator theorem [22] describing how the size of supernodes is evolving during the nested dissection process. According to our experiments, it is still interesting to have low-rank blocks at the end of the factorization, meaning that ranks remain lower than $\min \left(m_{C}, n_{C}\right) / 4$ (otherwise compression will not help), even if blocks received a large number of contributions. Thus, $r_{C^{\prime}}$ is often close or equal to $r_{C}$ and lower than $r_{C}+r_{A B}$ : the rank is often invariant applying a small contribution. So it is less expensive to use RRQR recompression (and operations are more suitable for performances). In terms of complexity, the recompression depends on the size of the target block $C$ and not on the size of the contribution blocks $A$ and $B$. As huge low-rank blocks are recompressed many times, it makes the Minimal Memory scenario slower than the dense version, but allows consequent memory savings. 
Table 1: Summary of the operation complexities when computing $C=C-A B^{t}$

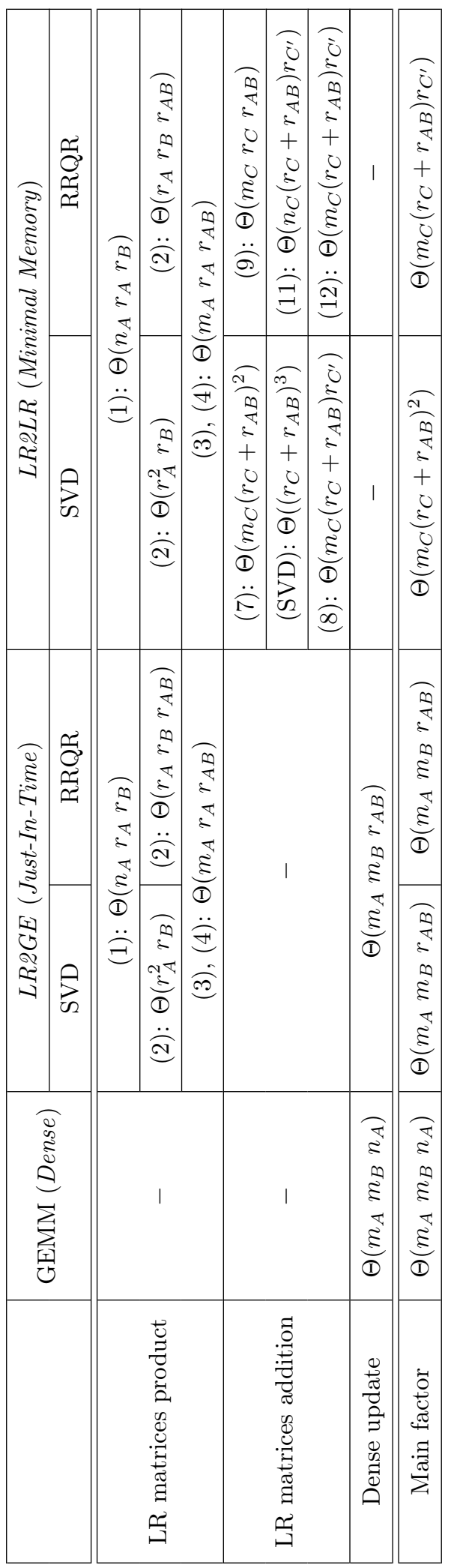


Finally, the main advantage of the Minimal Memory scenario is that it can drastically reduce the memory footprint of the solver, since it compresses the matrix before the factorization. Thus, the final structure of the dense factorized matrix is never allocated, and the low-rank structure needs to be maintained throughout the factorization process to lower the memory peak.

In order to overcome the issue of expensive low-rank additions, an idea would be to consider randomized techniques to allow an extend-add process depending on the size of contributing blocks and not on the size of the target block.

\section{Experiments}

Experiments were conducted on the Plafrim ${ }^{1}$ supercomputer, and more precisely on the miriel cluster. Each node is equipped with two INTEL Xeon E5-2680 v3 12-cores running at $2.50 \mathrm{GHz}$ and 128 GB of memory. The INTEL MKL 2016 is used for BLAS and SVD kernels. The RRQR kernel is coming from the BLR-MUMPS solver [16], and is an extension of the block rank-revealing QR factorization subroutines from LAPACK 3.6.0 (xGEQP3).

The PAStiX version used for our experiments is available on the public git repository ${ }^{2}$ as the tag papers/pdsec17. The multi-threaded version used is the static scheduling version presented in 23 .

For the initial ordering step, we used SCOTCH 20 5.1.11 with the configurable strategy string from PASTiX to set the minimal size of non separated sub-graphs, cmin, to 15 . We also set the frat parameter to 0.08 , meaning that columns aggregation is allowed by SCOTCH as long as the fill-in introduced does not exceed $8 \%$ of the original matrix.

In experiments, blocks that are larger than 256 are split in blocks of size at least 128 to create more parallelism while keeping sizes large enough. The same 128 criteria is used to defined the minimal width of the column blocks that are compressible. An additional limit on the minimal height to compress an off-diagonal block is set to 20 .

Experiments were computed on a set of 3D matrices extracted from The SuiteSparse Matrix Collection 24:

- Atmosmodj: atmospheric model (1 270432 dofs)

- Audi: structural problem (943 695 dofs)

- Hook: model of a steel hook (1 498023 dofs)

- Serena: gas reservoir simulation (1 391349 dofs)

- Geo1438: geomechanical model of earth (1 437960 dofs)

We also used 3D laplacian generators (7 points stencils), and defined lap120 as a laplacian of size $120^{3}$.

Note that when precision results are presented, we used the backward error on $b: \frac{\|A x-b\|_{2}}{\|b\|_{2}}$.

\subsection{SVD versus RRQR}

The first experiment studies the behavior of the two compression methods coupled both with Minimal Memory, and Just-In-Time scenario, on the matrix Atmosmodj. Table 2 presents the sequential timings of each operation of the numerical factorization with a tolerance of $10^{-8}$, as well as the memory used to store the final coefficient of the factorized matrix.

${ }^{1}$ https://plafrim.bordeaux.inria.fr

2 https://gitlab.inria.fr/solverstack/pastix 
Table 2: Costs distribution on the Atmosmodj matrix with $\tau=10^{-8}$

\begin{tabular}{|c|c|c|c|c|c|}
\hline & \multirow{2}{*}{ Dense } & \multicolumn{2}{|c|}{ Just-In-Time } & \multicolumn{2}{|c|}{ Minimal Memory } \\
\cline { 3 - 6 } & & RRQR & SVD & RRQR & SVD \\
\hline \hline Factorization time (s) & \multicolumn{5}{|l|}{} \\
\hline \hline Compression & - & 49.53 & 418.5 & 15.20 & 180.9 \\
\hline Block factorization & 0.9635 & 1.000 & 1.003 & 1.074 & 1.104 \\
\hline Panel solve & 15.80 & 6.970 & 6.526 & 11.16 & 6.946 \\
\hline LR product & - & 64.10 & 91.15 & 193.1 & 94.36 \\
\hline LR addition & - & - & - & 774.6 & 6523 \\
\hline Dense udpate & 418.7 & 47.94 & 47.03 & - & - \\
\hline \hline Total & 436 & 169 & 564 & 995 & 6806 \\
\hline \hline Solve time (s) & 2.43 & 1.54 & 1.8 & 2.22 & 1.29 \\
\hline \hline Factors final size (GB) & 15.9 & 7.4 & 6.86 & 11.4 & 6.76 \\
\hline
\end{tabular}

We can first notice that SVD compression kernels are much more time consuming than the RRQR kernels in both scenarios following the complexity study from Section 3 . Indeed, RRQR compression kernels stop the computations as soon as the rank is found which reduces by a large factor the complexity, and this reduction is reflected in the time-to-solution. However, the SVD allows, for a given tolerance, to get a better memory reduction in both scenarios.

Comparing the Minimal Memory and the Just-In-Time scenario, the compression time is minimized in the Minimal Memory scenario because the compression occurs on the initial blocks which hold more zeros and are lower ranks than once they have been updated. The time of the update addition, extend-add operation, becomes dominant in the Minimal Memory scenario, and even explodes when SVD is used. This is expected as the complexity depends on the largest blocks in the addition even for small contributions (see Section 3. In both scenarios, SVD kernels are able to keep the useful information and compress the final coefficients with similar rates, while the RRQR kernels are not as efficient to capture the information and to compress the blocks efficiently with the Minimal Memory scenario.

The diagonal blocks factorization time is invariant in the five strategies: the block sizes and kernels are identical. Panel solve, update product, and solve times are reduced in all low-rank configurations compared to the dense factorization and the timings follow the factors final size, since this size reflects the final ranks of the blocks.

To conclude, the Minimal Memory scenario is not able to compete with the original direct factorization due to the costly update addition. However, it reduced the memory peak of the solver to the factors final size. The Minimal Memory/RRQR offers a $25 \%$ memory reduction with a time to solution doubled in sequential. The Just-In-Time scenario competes with the original direct factorization, and divide by two the time-to-solution with RRQR kernels.

\subsection{Performance}

Figure 5 presents the overall performance achieved by the two low-rank scenarios with respect to the original version of the solver (where lower is better) on the previously introduced set of 6 matrices. All versions are multi-threaded implementations and use all the 24 cores of one node. The scheduling used is the PASTIX static scheduler developed for the original version, that is the only one available in the new development branch for now. This might have a negative impact on 


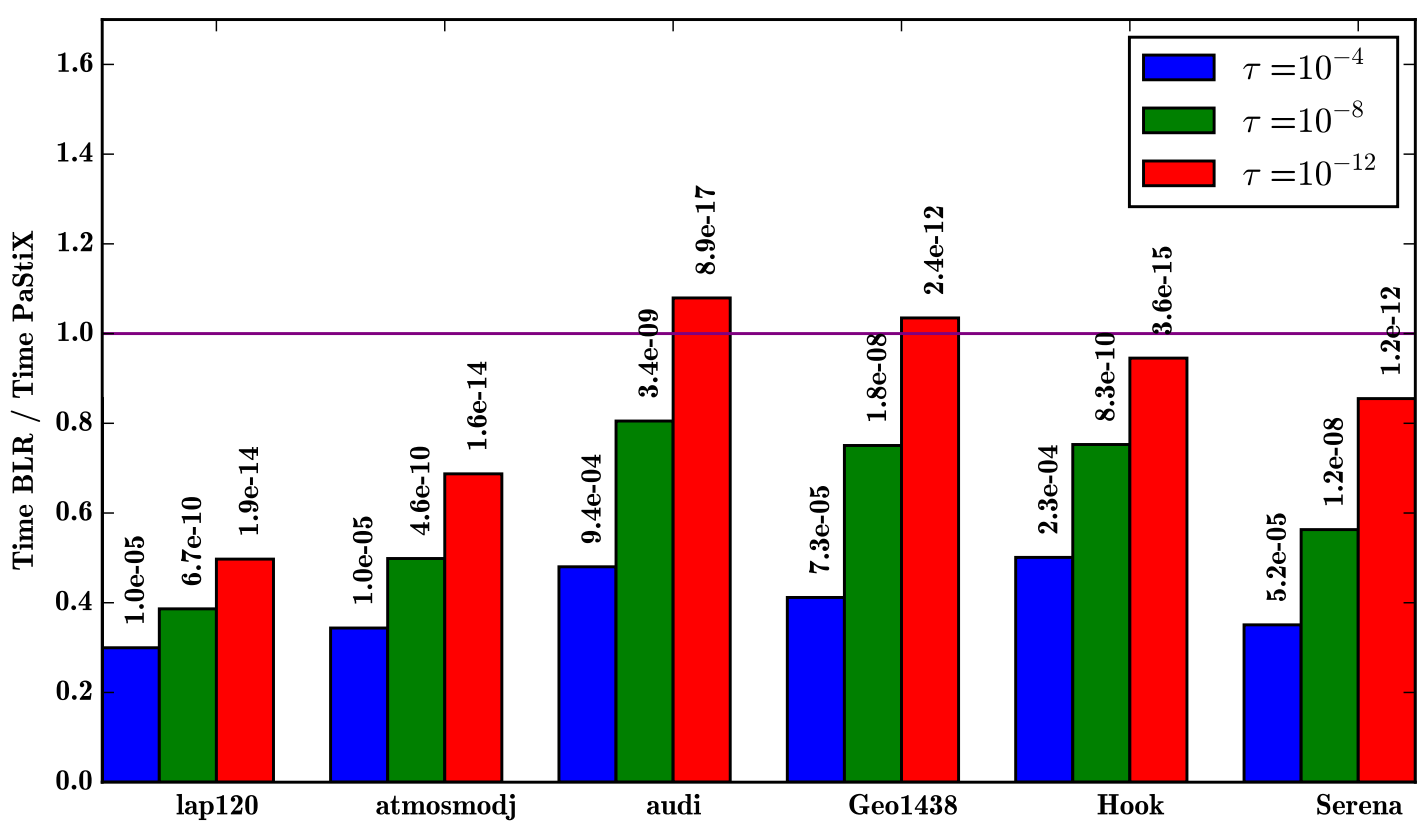

(a) Just-In-Time scenario using RRQR.

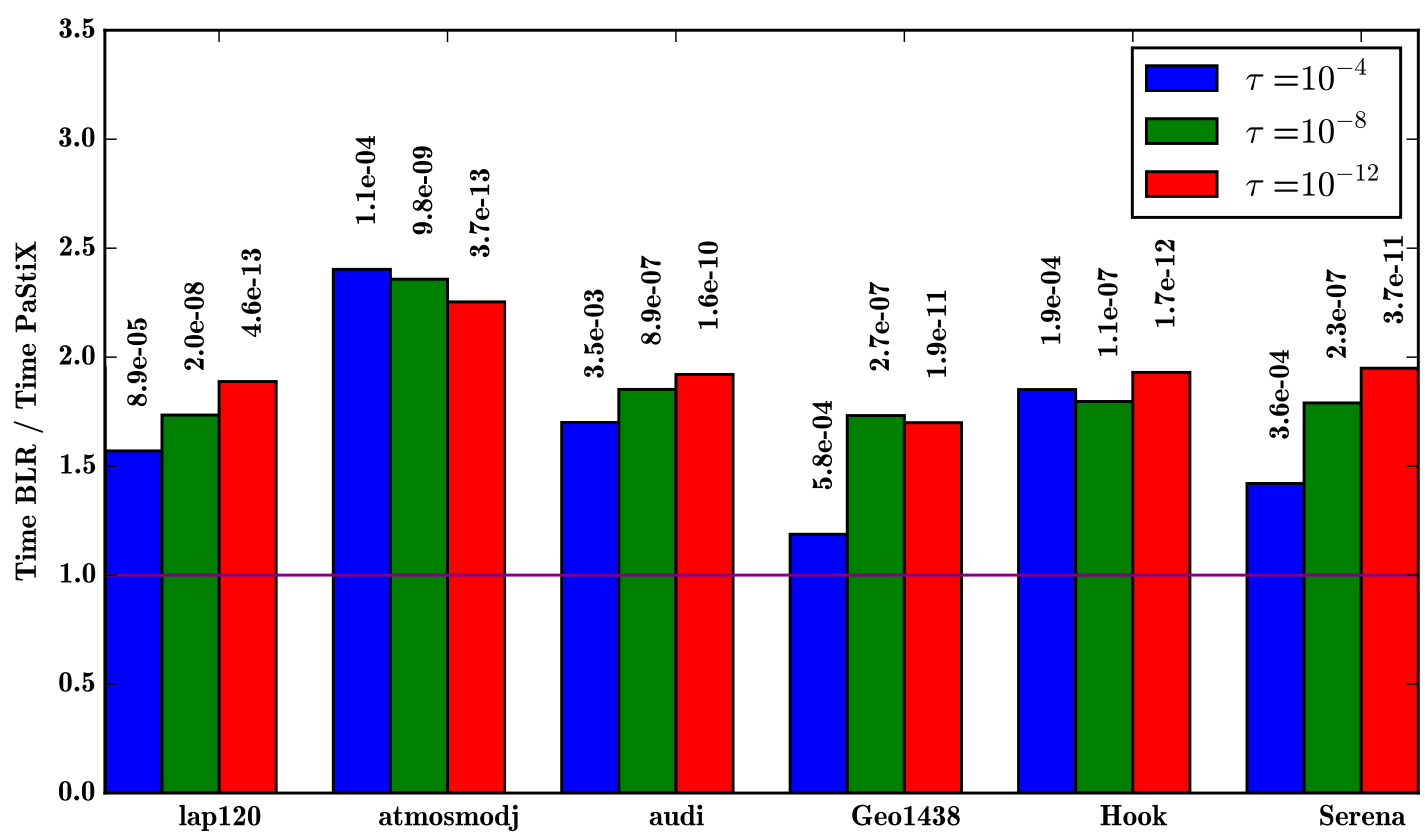

(b) Minimal Memory scenario using RRQR.

Figure 5: Performance of both strategies with 3 tolerance thresholds, backward error of the solution is printed on top of each bar. 
the low-rank implementations by creating a load imbalance. We study only the RRQR kernels as the SVD kernels have shown to be much slower. Three tolerance thresholds are studied for their impact on the time-to-solution, and the accuracy of the first residual of the solver, which went through one refinement step is shown with the backward errors printed on top of each bar.

Figure 5(a) shows that the Just-In-Time/RRQR scenario is able to reduce the time-tosolution in almost all cases of tolerance, and for all matrices which have a large spectrum of numerical properties. These results show that applications which requires low accuracy, as seismic for instance, can benefit up to a 3.3 speedup. Figure 5(b) shows that it is more difficult for the Minimal Memory/RRQR scenario to be competitive. The performance is always degraded with respect to the original PASTIX performance, with an average loss around a factor of 1.8, and the tolerance has a much lower impact than for the previous case.

For both scenarios, the backward error of the first solution is close to the entry tolerance. It is a little less accurate in the Minimal Memory scenario, because approximations are made earlier in the computations, and information is lost from the beginning. However, these results show that we are able to catch algebraically the information and forward it throughout the update process.

\subsection{Memory consumption}

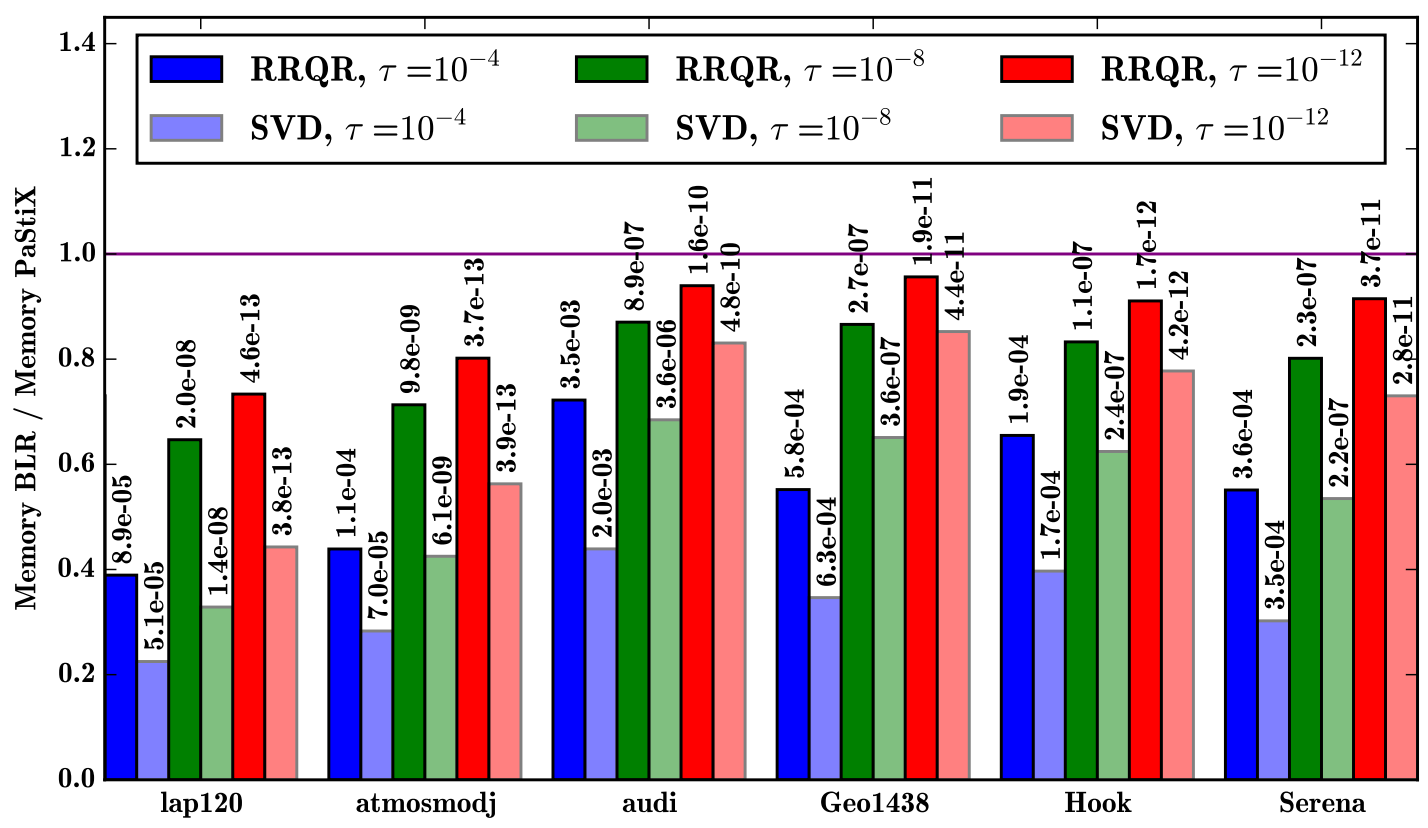

Figure 6: Memory peak for the Minimal Memory scenario with 3 tolerance thresholds and both SVD and RRQR kernels.

The Minimal Memory scenario is slower than the original solver, but it is a strategy that efficiently reduces the memory peak of the solver. Figure 6 presents the gain on the memory used to store the factors at the end of the factorization of the set of 6 matrices with respect to the block dense storage of PASTIX. In this figure, we also compare the memory gain of the SVD and 
RRQR kernels. We observe that in all cases, SVD provides a better compression rate by finding smaller ranks for a given matrix and a given tolerance. The quality of the first residual is also slightly better with the SVD kernels despite the smaller ranks. The second observation is that the smaller the tolerance $\left(10^{-12}\right)$, the larger the ranks and the memory consumption. However, the solver always presents a memory gain which can be more than $50 \%$ with larger tolerance $\left(10^{-4}\right)$.

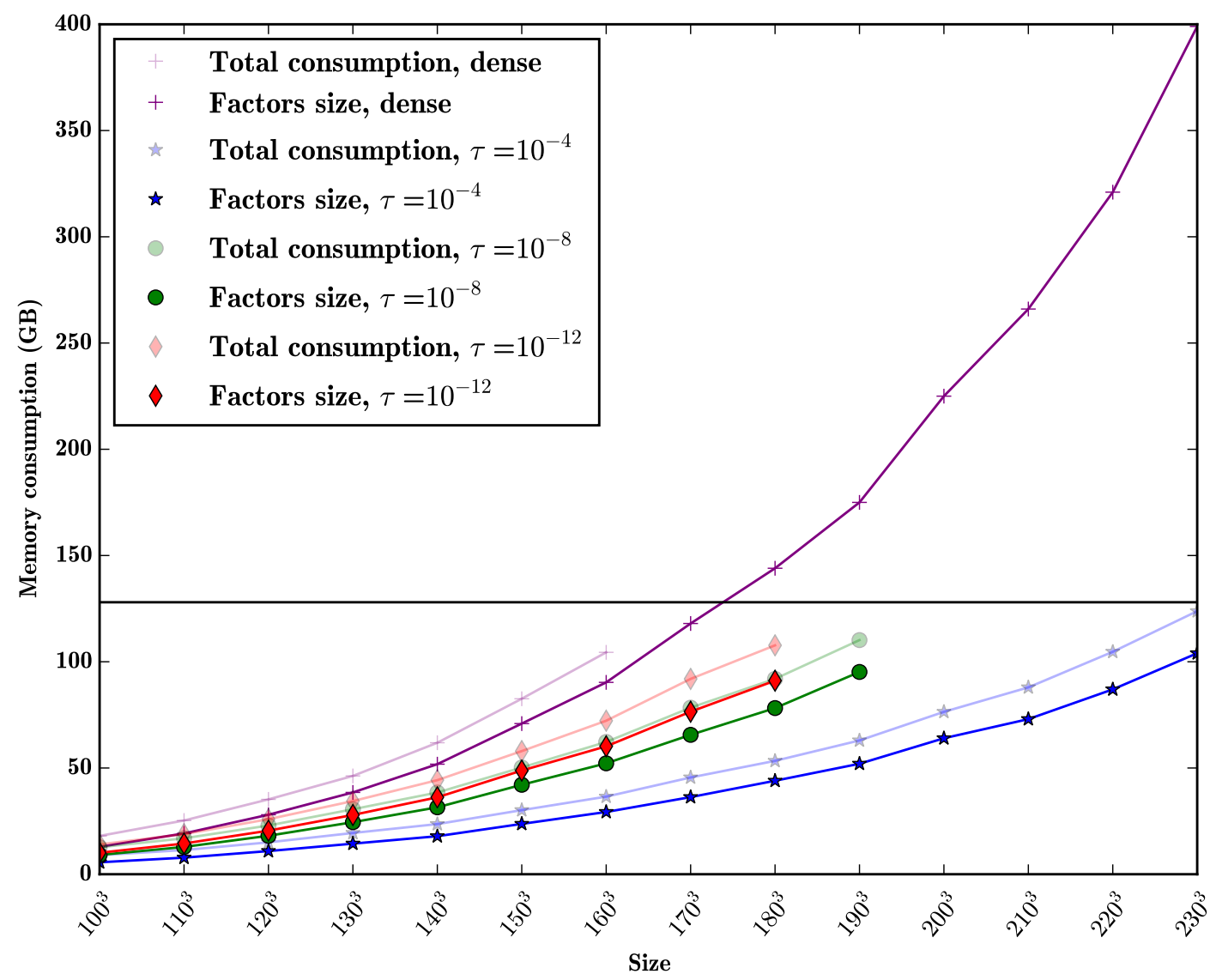

Figure 7: Memory scalability with 3 tolerance thresholds for the Minimal Memory/RRQR scenario when increasing the size of 3D Laplacians.

Figure 7 presents the evolution of the size of the factors as well as the full consumption of the solver (factors and management structures) on 3D Laplacians with an increasing size. The memory limit of the system is $128 \mathrm{~GB}$. The original version is limited on this system to a $3 \mathrm{D}$ Laplacian of 4 million unknowns, and the size of the factors quickly increase for larger sizes. With the Minimal Memory/RRQR scenario, we have now been able to run a 3D problem up to 12 million unknowns when relaxing the tolerance to $10^{-4}$.

The memory of the Just-In-Time scenario has not been studied, as long as in our supernodal approach, each supernode is fully allocated in a dense fashion in order to accumulate the update before being compressed. Thus, the memory peak corresponds to the totality of the factorized 
matrix structure without compression and is identical to the original version. To reduce this memory peak, a solution would be to modify the scheduler to a Left-Looking approach that would delay the allocation and the compression of the original blocks. However, it would need to be carefully implemented to keep a certain amount of parallelism in order to save both time and memory. A possible solution are the scheduling strategies presented in 25 to keep the memory consumption of the solver under a given limit.

\subsection{Convergence and numerical stability}

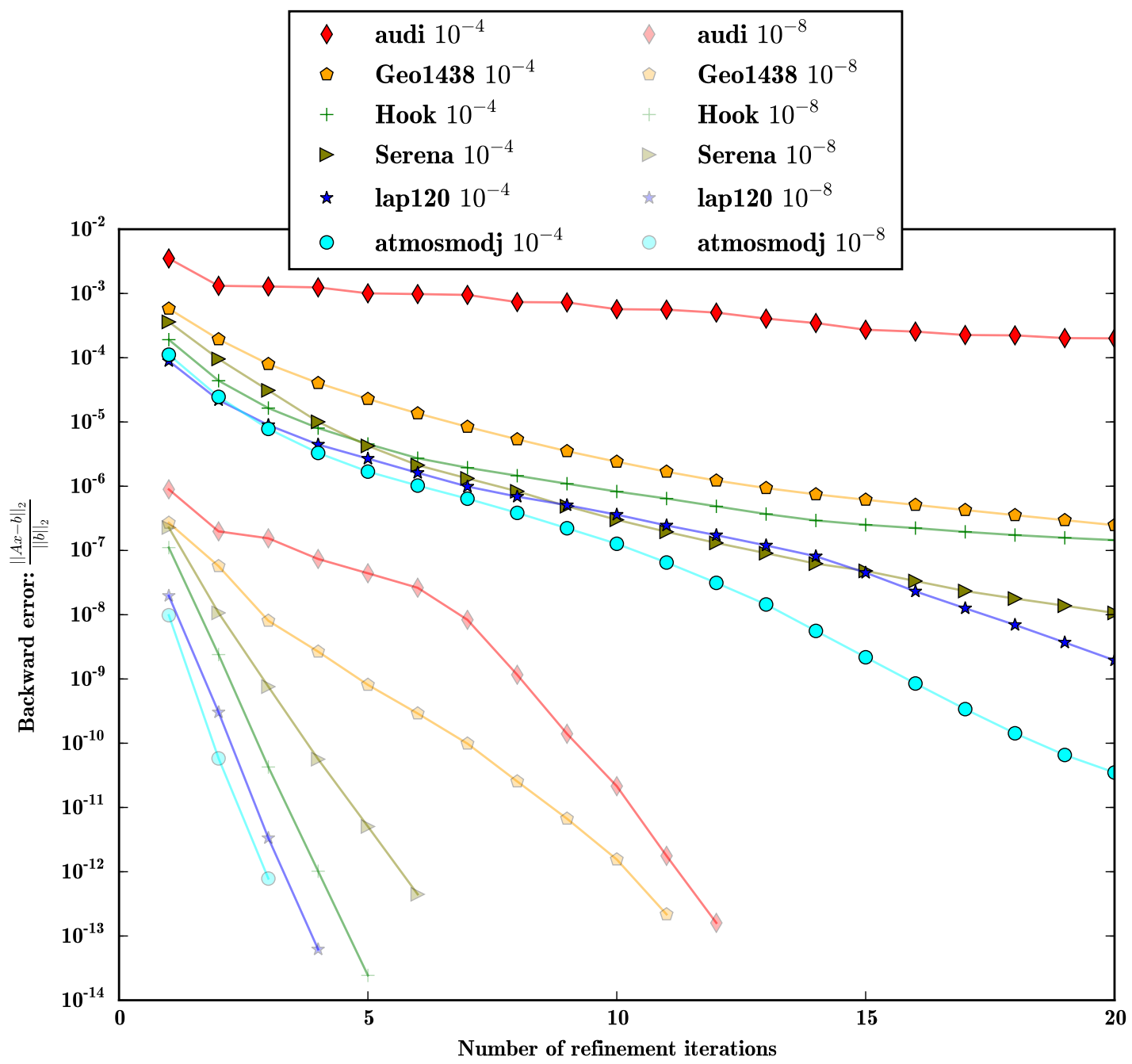

Figure 8: Convergence speed for the Minimal Memory/RRQR scenario with 2 tolerance thresholds.

Figure 8 presents the convergence of the iterative solver - GMRES for general matrices and Conjugate Gradient (CG) for SPD matrices - preconditioned with the low-rank factorization 
at tolerances of $10^{-4}$ and $10^{-8}$. The iterative solver is stopped after reaching 20 iterations or a backward error lower than $10^{-12}$.

With a tolerance of $10^{-8}$, only a few iterations are required to converge to the solution. Note that on the Audi and Geo1438 matrices, which are difficult to compress, a few more iterations are required to converge. With a larger tolerance $10^{-4}$, it is difficult to recover all the information lost during the compression, but this is enough to quickly get solutions at $10^{-6}$ or $10^{-8}$. Note that the iterative refinement process benefits from the compression, as the solve step, and is accelerated.

\section{Discussion}

In this section, we discuss the positioning of our solver with the closest related works and we give some limitations to extend this work to a hierarchical format.

Contrary to the approach studied in [1, we perform a symbolic block factorization. In their approach, as in our proposition, there is no fill-in between distinct branches of the elimination tree. However, contributions of a supernode to its ancestors are considered as full, in the sense that all structural zeros are included to generate the low-rank representation. Thus, they do not have extend-add ( $L R 2 L R$ ) operation between low-rank blocks of different sizes, but the memory consumption is higher because some structural zeros are not managed.

A dense BLR solver was designed by Livermore Software Technology Corporation [15]. In this work, the full matrix is compressed at the beginning and operations between low-rank blocks are performed. This approach is similar to our Minimal Memory scenario in the context of dense matrices. Due to this restriction, the extend-add process concerns low-rank matrices of the same size, without zeros padding. Thus, the LR2LR operation is less costly than the dense update in their context.

A BLR multifrontal sparse direct solver was designed for the MUMPS solver. The strategy is described in [16] and a theoretical study of the complexity of the solver is presented in $[26$. In this work, contribution blocks are not compressed. When a front is eliminated, different strategies are proposed to enhance the time-to-solution. Our scenario Just-In-Time is close to their FCSU (Factor, Compress, Solve, Update) strategy. The LUAR (Low-Rank Update Accumulation with Recompression) groups together multiple low-rank products to exploit the memory locality during the product recompression process. This could be similarly used in the Just-In-Time, but would implies larger ranks in the extend-add operations of the Minimal Memory. The CUFS (Compress, Update, Factor, Solve) is the strategy closest to our Minimal Memory scenario. However, only a dense front is fully compressed before being eliminated: contributing blocks are not compressed and low-rank operations occur within a dense matrix, similarly to the previous work from LSTC. If the time-to-solution is better with BLR-MUMPS, there is more room for memory savings in our approach.

With the aim of extending our solver to hierarchical compression schemes, such as $\mathcal{H}$, HSS, or HODLR, we consider graphs issued from finite element meshes coming from real-life simulations of $3 \mathrm{D}$ physical problems. From a theoretical point of view, the majority of these graphs have a bounded degree and thus good separators respecting the separator theorems $[22$ can be built. For a $n$-vertices mesh, the time complexity of a direct solver is in $\Theta\left(n^{2}\right)$, and we expect to build a low-rank solver requiring $\Theta\left(n^{\frac{4}{3}}\right)$ operations. For the memory requirements, the direct approach leads to an overall storage in $\Theta\left(n^{\frac{4}{3}}\right)$, while we target a $\Theta(n \log (n))$ complexity. Let us consider the last separator of size $\Theta\left(n^{\frac{2}{3}}\right)$ for a 3D mesh, and one of the largest low-rank block of this separator. They are asymptotically the same size. Previous studies have shown that such a block may have a rank in order of $\Theta\left(n^{\frac{1}{3}}\right)$. 
For the Minimal Memory scenario, we have seen that the time-to-solution is longer than the dense version. As low-rank blocks become larger in the hierarchy, it will be even worse than the solution we developed. For the Just-In-Time scenario, maintaining such a block in a dense form before compressing block requires $\Theta\left(n^{\frac{4}{3}}\right)$ memory and does not satisfy the memory complexity we target. It also means that a compromise between Minimal Memory and Just-In-Time strategies using a Left-Looking approach might not be a relevant solution. Currently, no sparse solver is able to perform efficiently the extend-add operations using compression techniques such as SVD or RRQR, and it is still an open problem.

\section{Conclusion}

We presented a new Block Low-Rank sparse solver that combines an existing sparse direct solver PASTIX, and low-rank compression kernels. This solver reduces the memory consumption and/or the time-to-solution depending on the scenario. Two scenarios were developed. Minimal Memory saves memory up to a factor of 2.6 using RRQR kernels, with a time overhead that is limited to 2.4 despite the higher complexity. Large problems that could not fit into memory when the original solver was used can now be solved thanks to the lower memory requirements, especially when low accuracy solutions and/or large number of right hand sides are involved.

Just-In-Time reduces both the time-to-solution by a factor up to 3.3, and the memory requirements of the final factorized matrix with similar factors to Minimal Memory. However, with the actual scheduling strategy, this gain is not reflected on the memory peak.

Two compression kernels, SVD and RRQR, were studied and compared. We have shown that, for a given tolerance, both approaches provide correct solutions with the expected accuracy, and that RRQR despite larger ranks provides faster kernels. In addition, we demonstrated that the solver can be used either as a low-tolerance direct solver, or as a good preconditioner for iterative methods, that will require only a few iterations before reaching the machine precision.

In the future, new kernel families, such as RRQR with randomization techniques, will be studied in terms of accuracy and stability in the context of a supernodal solver. To further improve the performance of Minimal Memory and close up the gap with the original solver, aggregation techniques on small contributions will also be studied. This will lead to the extension of this work to hierarchical compression in large supernodes that could further reduce the memory footprint, and the solver complexity.

Regarding Just-In-Time, future work is focused on studying smart scheduling strategies that combines Right-Looking and a Left-Looking approach in order to find a good compromise between memory and parallelism for the targeted architecture. This will follow up recent work on applying parallel runtime systems 23 to the PAStiX solver.

\section{Acknowledgments}

This material is based upon work supported by the DGA under a DGA/Inria grant. Experiments presented in this paper were carried out using the PLAFRIM experimental platform.

\section{References}

[1] W. Hackbusch, Hierarchical Matrices: Algorithms and Analysis. Springer Series in Computational Mathematics, 2015, vol. 49. 
[2] L. Grasedyck, R. Kriemann, and S. Le Borne, "Parallel black box $\mathcal{H}$-LU preconditioning for elliptic boundary value problems," Computing and Visualization in Science, vol. 11, no. 4-6, pp. 273-291, 2008.

[3] L. Grasedyck, W. Hackbusch, and R. Kriemann, "Performance of H-LU preconditioning for sparse matrices," Computational methods in applied mathematics, vol. 8, no. 4, pp. 336-349, 2008 .

[4] R. Kriemann, "H-LU factorization on many-core systems," Computing and Visualization in Science, vol. 16, no. 3, pp. 105-117, 2013.

[5] A. Aminfar, S. Ambikasaran, and E. Darve, "A fast block low-rank dense solver with applications to finite-element matrices," Journal of Computational Physics, vol. 304, pp. 170-188, 2016.

[6] A. Aminfar and E. Darve, "A fast, memory efficient and robust sparse preconditioner based on a multifrontal approach with applications to finite-element matrices," International Journal for Numerical Methods in Engineering, 2016.

[7] J. N. Chadwick and D. S. Bindel, "An Efficient Solver for Sparse Linear Systems Based on Rank-Structured Cholesky Factorization," CoRR, vol. abs/1507.05593, 2015.

[8] J. Xia, S. Chandrasekaran, M. Gu, and X. Li, "Superfast Multifrontal Method For Large Structured Linear Systems of Equations," Siam Journal on Matrix Analysis and Applications, vol. 31, p. 1382-1411, 2009.

[9] S. Wang, X. S. Li, F.-H. Rouet, J. Xia, and M. V. De Hoop, "A Parallel Geometric Multifrontal Solver Using Hierarchically Semiseparable Structure," ACM Trans. Math. Softw., vol. 42, no. 3, pp. 21:1-21:21, May 2016.

[10] P. Ghysels, X. S. Li, F.-H. Rouet, S. Williams, and A. Napov, "An Efficient Multicore Implementation of a Novel HSS-Structured Multifrontal Solver Using Randomized Sampling," SIAM Journal on Scientific Computing, vol. 38, no. 5, pp. S358-S384, 2016.

[11] H. Pouransari, P. Coulier, and E. Darve, "Fast hierarchical solvers for sparse matrices using extended sparsification and low-rank approximation," arXiv preprint arXiv:1510.07363v3, 2015 .

[12] K. Yang, H. Pouransari, and E. Darve, "Sparse hierarchical solvers with guaranteed convergence," arXiv preprint arXiv:1611.03189, 2016.

[13] K. L. Ho and L. Ying, "Hierarchical interpolative factorization for elliptic operators: differential equations," Communications on Pure and Applied Mathematics, 2015.

[14] D. A. Sushnikova and I. V. Oseledets, "“Compress and eliminate" solver for symmetric positive definite sparse matrices," arXiv preprint arXiv:1603.09133v3, 2016.

[15] J. Anton, C. Ashcraft, and C. Weisbecker, "A Block Low-Rank Multithreaded Factorization for Dense BEM Operators," in SIAM Conference on Parallel Processing for Scientific Computing (SIAM PP 2016), Paris, France, Apr. 2016.

[16] P. Amestoy, C. Ashcraft, O. Boiteau, A. Buttari, J.-Y. L'Excellent, and C. Weisbecker, "Improving Multifrontal Methods by Means of Block Low-Rank Representations," SIAM Journal on Scientific Computing, vol. 37, no. 3, pp. A1451-A1474, 2015. 
[17] P. Hénon, P. Ramet, and J. Roman, "PaStiX: A High-Performance Parallel Direct Solver for Sparse Symmetric Definite Systems," Parallel Computing, vol. 28, no. 2, pp. 301-321, Jan. 2002.

[18] A. George, "Nested dissection of a regular finite element mesh," SIAM Journal on Numerical Analysis, vol. 10, no. 2, pp. 345-363, 1973.

[19] G. Karypis and V. Kumar, "METIS: A software package for partitioning unstructured graphs, partitioning meshes, and computing fill-reducing orderings of sparse matrices," 1995.

[20] F. Pellegrini, "Scotch and libScotch 5.1 User's Guide," Aug. 2008, user's manual, 127 pages.

[21] G. Pichon, M. Faverge, P. Ramet, and J. Roman, "Reordering strategy for blocking optimization in sparse linear solvers," Inria, Research Report, to appear in SIAM Journal on Matrix Analysis and Applications RR-8860, Feb. 2016.

[22] R. J. Lipton and R. E. Tarjan, "A separator theorem for planar graphs," SIAM Journal on Applied Mathematics, vol. 36, pp. 177-189, 1979.

[23] X. Lacoste, "Scheduling and memory optimizations for sparse direct solver on multicore/multi-gpu cluster systems," Ph.D. dissertation, Bordeaux University, Talence, France, Feb. 2015.

[24] T. A. Davis and Y. Hu, "The University of Florida sparse matrix collection," ACM Trans. Math. Softw., vol. 38, no. 1, pp. 1:1-1:25, Dec. 2011.

[25] E. Agullo, P. R. Amestoy, A. Buttari, A. Guermouche, J. L'Excellent, and F. Rouet, "Robust Memory-Aware Mappings for Parallel Multifrontal Factorizations," SIAM Journal on Scientific Computing, vol. 38, no. 3, 2016.

[26] P. Amestoy, A. Buttari, J.-Y. L'Excellent, and T. Mary, "On the Complexity of the Block Low-Rank Multifrontal Factorization," IRIT, Research Report RT-2016-03-FR, May 2016. 


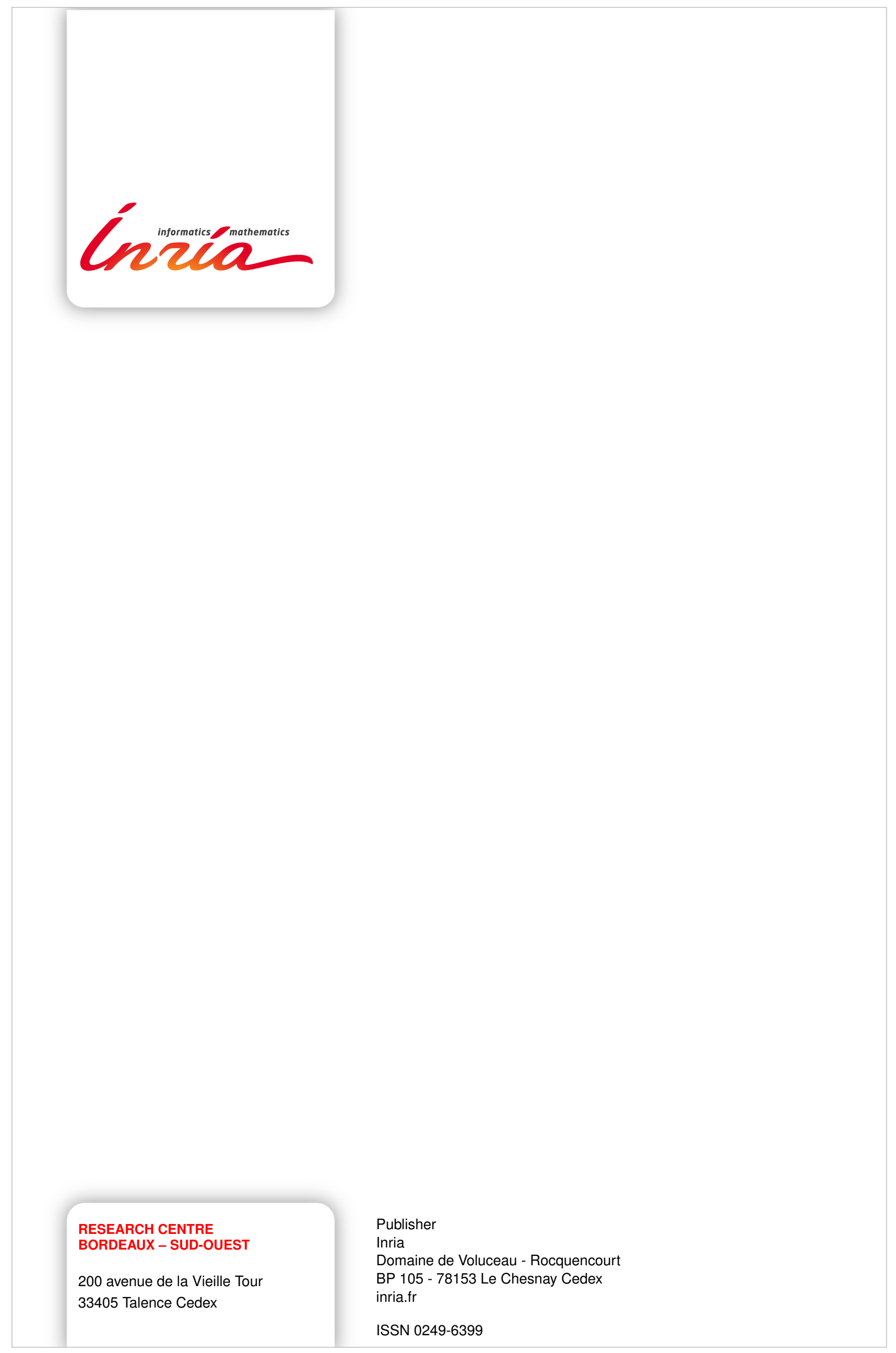

